\title{
Satisfacción de las madres de niños hospitalizados frente a los cuidados de enfermería en el servicio de pediatría del Hospital Regional de Pucallpa, 2020
}

Satisfaction of mothers of hospitalized children with nursing care in the pediatric service of the Regional Hospital of Pucallpa, 2020

Satisfação das mães de crianças hospitalizadas com cuidados de enfermagem no serviço pediátrico do Hospital Regional de Pucallpa, 2020

\section{ARTÍCULO GENERAL}

Nicolás Magno Fretel Quiroz

nicolasfretel@gmail.com

Docente Contratado de la

Carrera de Enfermería de la UNAMAD

\begin{tabular}{c|c}
$\begin{array}{c}\text { Holger Alex Aranciaga } \\
\text { Campos }\end{array}$ & $\begin{array}{c}\text { Roger Habraham } \\
\text { Tapia Trujillo } \\
\text { holger1964campos@gmail.com } \\
\text { rogertapia15@gmail.com }\end{array}$ \\
$\begin{array}{c}\text { Docente Asociado de la } \\
\text { Facultad de Enfermería de } \\
\text { la UNHEVAL. }\end{array}$ & $\begin{array}{c}\text { Investigador } \\
\text { Independiente }\end{array}$ \\
\end{tabular}

Reyna Isabel

Bastos Vásquez reynaisabel@gmail.com

Lic. Enf. Hospital

Regional de Pucallpa

Recibido 05 de Febrero 2021 | Arbitrado y aceptado 19 de Abril 2021 | Publicado 28 de Julio 2021

\section{RESUMEN}

El objetivo del estudio fue determinar el nivel de satisfacción de las madres de niños hospitalizados frente a los cuidados de Enfermería en el Servicio de Pediatría del Hospital Regional de Pucallpa, 2020. Metodología; corresponde a un diseño no experimental; descriptivo, transversal y prospectivo; población de 62 madres de niños hospitalizados en el servicio de Pediatría del Hospital Regional de Pucallpa durante los meses de noviembre y diciembre de 2020; como técnica se utilizó la encuesta y como instrumento un cuestionario compuesto de 24 item's. Resultados, el $51.6 \%$ (32) de las madres encuestadas expresan nivel de satisfacción intermedia; el $25.8 \%$ (16) de las madres encuestadas expresan nivel de satisfacción completa; y el $22.6 \%$ (14) de las madres encuestadas expresan insatisfacción. Conclusiones; más de la mitad de las madres encuestadas expresan nivel de satisfacción intermedia con tendencia al nivel de satisfacción completa, pero aún persiste un considerable porcentaje que expresan insatisfacción. La satisfacción, en la dimensión elementos tangibles, en el $59.7 \%$ expresaron satisfacción intermedia; en la dimensión fiabilidad, el $56.5 \%$ expresaron satisfacción intermedia; en la dimensión capacidad de respuesta, el $53.2 \%$ expresaron satisfacción intermedia; en la dimensión seguridad, el $56.5 \%$ expresaron satisfacción intermedia; en la dimensión empatía, el $59.7 \%$ expresaron satisfacción intermedia.

Palabras clave; satisfacción, cuidado de enfermería.

\section{ABSTRACT}

The objective of the study was to determine the level of satisfaction of mothers of hospitalized children with Nursing care in the Pediatric Service of the Hospital Regional de Pucallpa, 2020. Methodology; corresponds to a non-experimental design; descriptive, transversal and prospective; population of 62 mothers of children hospitalized in the Pediatrics service of the Regional Hospital of Pucallpa during the months of November and December 2020; The survey was used as a technique and a questionnaire made up of 24 items as an instrument. Results, $51.6 \%$ (32) of the mothers surveyed express an intermediate level of satisfaction; $25.8 \%$ (16) of the mothers surveyed express a level of complete satisfaction; and $22.6 \%$ (14) of the mothers surveyed express dissatisfaction. Conclusions; More than half of the mothers surveyed express an intermediate level of satisfaction with a trend towards a level of complete satisfaction, but there is still a considerable percentage who express dissatisfaction. Satisfaction, in the tangible elements dimension, $59.7 \%$ expressed intermediate satisfaction; In the reliability dimension, $56.5 \%$ expressed intermediate satisfaction; In the response capacity dimension, $53.2 \%$ expressed intermediate satisfaction; In the security dimension, $56.5 \%$ expressed intermediate satisfaction; In the empathy dimension, $59.7 \%$ expressed intermediate satisfaction.

Keywords; satisfaction, nursing care.

\section{RESUMO}

O objetivo do estudo foi determinar o nível de satisfação das mães de crianças hospitalizadas com os cuidados de enfermagem no Serviço de Pediatria do Hospital Regional de Pucallpa, 2020. Metodologia; corresponde a um desenho não experimental; descritivo, transversal $\mathrm{e}$ prospectivo; população de 62 mães de crianças internadas no serviço de Pediatria do Hospital Regional de Pucallpa nos meses de novembro e dezembro de 2020; A pesquisa foi utilizada como técnica e um questionário composto por 24 itens como instrumento. Resultados, $51,6 \%$ (32) das mães pesquisadas expressam um nível intermediário de satisfação; $25,8 \%$ (16) das mães pesquisadas expressam um nível de satisfação total; e $22,6 \%$ (14) das mães pesquisadas expressam insatisfação. Conclusões; Mais da metade das mães pesquisadas expressa um nível intermediário de satisfação com tendência a um nível de satisfação total, mas ainda há um percentual considerável que expressa insatisfação. Satisfação, na dimensão dos elementos tangíveis, $59,7 \%$ expressaram satisfação intermediária; $\mathrm{Na}$ dimensão confiabilidade, $\quad 56,5 \%$ expressaram satisfação intermediária; $\mathrm{Na}$ dimensão capacidade de resposta, $53,2 \%$ expressaram satisfação intermediária; $\mathrm{Na}$ dimensão segurança, $56,5 \%$ expressaram satisfação intermediária; $\mathrm{Na}$ dimensão empatia, $59,7 \%$ expressaram satisfação intermediária.

Palavras chave; satisfação, cuidados de enfermagem. 


\section{INTRODUCCIÓN}

El concepto de satisfacción del usuario se basa en la diferencia entre las expectativas del usuario y la percepción de los servicios que ha recibido; de esta manera, las percepciones subjetivas acompañadas de las expectativas previas configuran la expresión de la calidad del servicio. Según la literatura consultada importa destacar, desde una perspectiva multidimensional, el modelo propuesto por SERVQUAL, han identificado cinco dimensiones que los pacientes utilizan para juzgar el servicio: Elementos Tangibles, referido a los ambientes adecuados y acondicionados, ambientes en condiciones óptimas, instalaciones sanitarias adecuadas, equipamiento, ambientes limpios y cómodos, personal uniformado y con apariencia limpia. Fiabilidad, considera: las señalizaciones para orientar, brindan materiales impresos, se preocupan por los problemas y necesidades del usuario, expresión de opinión; capacidad de respuesta, considera: el tiempo necesario para contestar sus dudas o preguntas sobre su problema de salud, que le expliquen los procedimientos y la administración el tratamiento; seguridad, considera, inspira confianza, sentimiento de seguridad, seguridad en la administración del tratamiento o procedimiento; Empatía, considera trato con amabilidad, atención inmediata, el personal muestra interés por las necesidades individuales de los pacientes.

En los últimos años, el estudio de satisfacción de los usuarios, con respecto a los servicios sanitarios, se ha convertido en un instrumento de valor creciente, tomando ejemplos como la encuesta de satisfacción de usuarios con los servicios de atención primaria realizada por el centro de investigaciones sociológicas aplicada durante varios años. El hecho de que se acepte que la satisfacción del usuario es un resultado importante del trabajo realizado por los profesionales justifica que esta se haya incorporado como una medida de calidad. El análisis de la satisfacción también se está utilizando como instrumento para legitimar las diferentes reformas sanitarias, ya que la opinión del usuario puede utilizarse sistemáticamente para mejorar la organización de los servicios.

\section{Hipótesis}


Por las características del estudio desarrollado, de nivel descriptivo, y en concordancia a lo publicado por el autor Hernández R. en su texto metodología de la investigación, los estudios descriptivos no ameritan formular hipótesis.

\section{METODOLOGÍA}

El estudio respondió al nivel descriptivo, ya que la variable en estudio se midió de manera individual para luego elaborar tablas de frecuencia mediante el uso de la estadística descriptiva.

Tipo: Transversal, porque la recolección de los datos se desarrolló en un solo momento. Prospectivo, porque los datos se generaron luego de iniciado la investigación.

Diseño no experimental por que la variable fue estudiada en su contexto natural, sin ninguna manipulación intencional de la responsable del proceso de investigación. La población muestral, estuvo constituida por todas las madres de niños hospitalizados en el servicio de Pediatría del Hospital Regional de Pucallpa durante los meses de noviembre y diciembre de 2020, haciendo un total de 62 madres.

Técnica, se hizo uso de técnica de la encuesta.

Instrumento, el instrumento utilizado fue un cuestionario que tiene 24 items con 3 alternativas de respuesta (Siempre $=2$, A veces $=1$, y Nunca $=0$ ), mediante el cual se estableció las siguientes categorías:

- Satisfacción completa

- Satisfacción intermedia:

- Insatisfacción: 
RESULTADOS

Los resultados obtenidos son los siguientes:

\section{Tabla 1}

Satisfacción de las madres de niños hospitalizados frente a los cuidados de

Enfermería en el Servicio de Pediatría del Hospital Regional de

Pucallpa, 2020

\begin{tabular}{ccc}
\hline Nivel de Satisfacción & $\mathrm{N}$ & $\%$ \\
\hline Satisfacción completa: & 16 & 25.8 \\
Satisfacción intermedia: & 32 & 51.6 \\
Insatisfacción: & 14 & 22.6 \\
Total & 62 & 100.0
\end{tabular}

Fuente: Cuestionario aplicado a las madres de niños Hospitalizados en el servicio de pediatría del HRP, 2020

Del total de madres encuestadas (62), el 51.6\% (32) de ellas expresa nivel de satisfacción intermedia; el 25.8\% (16) expresa nivel de satisfacción completa; y el $22.6 \%$ (14) de ellas expresa insatisfacción.

El resultado obtenido fue diferente a los hallazgos publicado por Sango B., en Ecuador el 2014, quien en su estudio titulado Grado de satisfacción de la madre por la atención brindada por la Enfermera con los pacientes hospitalizados, Hospital Provincial General Latacunga en el servicio de pediatría, llegaron a las siguientes conclusiones: El grado de satisfacción de las madres con respecto a la atención de enfermería fue satisfactoria en un $43 \%$. (8)

Así mismo, los resultados difieren de los hallazgos de Coronado A., y Vásquez K., en su estudio sobre el Nivel de satisfacción de la madre sobre el cuidado brindado por la enfermera al niño hospitalizado en Pediatría del Hospital Regional Lambayeque, 2017, indicaron que el $83 \%$ estuvieron muy satisfechas, $11 \%$ estuvieron satisfechas y $6 \%$ de madres estuvieron insatisfechas con el cuidado brindado. (11) 
Pero, son similares a los hallazgos de Velásquez D., en el estudio sobre Satisfacción de las madres de niños hospitalizados en pediatría sobre la atención de Enfermería del Hospital Manuel Nuñez Butrón, donde el 55\% de las madres presentan satisfacción intermedia, seguida del $35 \%$ de satisfacción completa y finalmente un $10 \%$ de las madres manifiestan insatisfacción. (13)

\section{Tabla 2}

Satisfacción, en la dimensión elementos tangibles, de las madres de niños hospitalizados frente a los cuidados de Enfermería en el Servicio de Pediatría del Hospital Regional de Pucallpa,

\begin{tabular}{ccc}
\hline $\begin{array}{c}\text { Nivel de Satisfacción en la dimensión } \\
\text { Elementos tangibles }\end{array}$ & $\mathrm{N}$ & $\%$ \\
\hline Satisfacción completa: & 13 & 20.9 \\
Satisfacción intermedia: & 37 & 59.7 \\
Insatisfacción: & 12 & 19.4 \\
Total & 62 & 100.0
\end{tabular}

Fuente: Cuestionario aplicado a las madres de niños Hospitalizados en el servicio de pediatría del HRP, 2020

Del total de madres encuestadas (62), el 59.7\% (37) de ellas expresan satisfacción intermedia; el 20.9\% (13) expresan satisfacción completa; y el 19.4\% (12) de ellas expresan insatisfacción.

Resultados similares a los hallazgos publicado por Velásquez D., en el estudio sobre Satisfacción de las madres de niños hospitalizados en pediatría sobre la atención de Enfermería del Hospital Manuel Nuñez Butrón, donde, en cuanto a la Dimensión de Elementos Tangibles el 55\% de las madres muestran satisfacción intermedia, seguido con el 35\% de satisfacción completa, y finalmente un 10\% de insatisfacción. (13) 
Tabla 3

Satisfacción, en la dimensión fiabilidad, de las madres de niños hospitalizados frente a los cuidados de Enfermería en el Servicio de Pediatría del Hospital Regional de Pucallpa, 2020

\begin{tabular}{ccc}
\hline Nivel de Satisfacción en la dimensión fiabilidad & $\mathrm{N}$ & $\%$ \\
\hline Satisfacción completa: & 16 & 25.8 \\
Satisfacción intermedia: & 35 & 56.5 \\
Insatisfacción: & 11 & 17.7 \\
Total & 62 & 100.0
\end{tabular}

Fuente: Cuestionario aplicado a las madres de niños Hospitalizados en el servicio de pediatría del HRP, 2020

Del total de madres encuestadas (62), el 56.5\% (35) de ellas expresan satisfacción intermedia; el 25.8\% (16) expresan satisfacción completa; y el 17.7\% (11) de ellas expresan insatisfacción.

Resultados diferentes a los hallazgos publicado por Velásquez D., en el estudio sobre Satisfacción de las madres de niños hospitalizados en pediatría sobre la atención de Enfermería del Hospital Manuel Nuñez Butrón, donde, en cuanto a la Dimensión de Fiabilidad, el 55\% de las madres muestran insatisfacción, seguido del 25\% de satisfacción intermedia y finalmente un $20 \%$ de satisfacción completa. (13) 
Nicolás Magno Fretel, Eldalaine Torres Vargas, Holger Alex Aranciaga, Roger Habraham Tapia, Reyna Isabel Bastos Vásquez

Tabla 4

Satisfacción, en la dimensión capacidad de respuesta, de las madres de niños hospitalizados frente a los cuidados de Enfermería en el Servicio de Pediatría del Hospital Regional de Pucallpa, 2020

\begin{tabular}{ccc}
\hline $\begin{array}{c}\text { Nivel de Satisfacción en la dimensión capacidad de } \\
\text { respuesta }\end{array}$ & $\mathrm{N}$ & $\%$ \\
\hline Satisfacción completa: & 17 & 27.4 \\
Satisfacción intermedia: & 33 & 53.2 \\
Insatisfacción: & 12 & 19.4 \\
Total & 62 & 100.0
\end{tabular}

Fuente: Cuestionario aplicado a las madres de niños Hospitalizados en el servicio de pediatría del HRP, 2020

Del total de madres encuestadas (62), el 53.2\% (33) de ellas expresan satisfacción intermedia; el 27.4\% (17) expresan satisfacción completa; y el 19.4\% (12) de ellas expresan insatisfacción.

Los resultados son similares a los hallazgos de Velásquez D., en el estudio sobre Satisfacción de las madres de niños hospitalizados en pediatría sobre la atención de Enfermería del Hospital Manuel Nuñez Butrón, donde, en cuanto a la Dimensión de Capacidad de Respuesta, un 50\% de las madres muestran satisfacción intermedia, seguido del $40 \%$ de satisfacción y finalmente un 10\% de insatisfacción. (13) 


\section{Tabla 5}

Satisfacción, en la dimensión seguridad, de las madres de niños hospitalizados frente a los cuidados de Enfermería en el Servicio de Pediatría del Hospital Regional de Pucallpa, 2020

\begin{tabular}{ccc}
\hline Nivel de Satisfacción en la dimensión seguridad & $\mathrm{N}$ & $\%$ \\
\hline Satisfacción completa: & 16 & 25.8 \\
Satisfacción intermedia: & 35 & 56.5 \\
Insatisfacción: & 11 & 17.7 \\
Total & 62 & 100.0
\end{tabular}

Fuente: Cuestionario aplicado a las madres de niños Hospitalizados en el servicio de pediatría del HRP, 2020

Del total de madres encuestadas (62), el 56.5\% (35) de ellas expresan satisfacción intermedia; el 25.8\% (16) expresan satisfacción completa; y el 17.7\% (11) de ellas expresan insatisfacción.

Los resultados difieren de los hallazgos de Velásquez D., en el estudio sobre Satisfacción de las madres de niños hospitalizados en pediatría sobre la atención de Enfermería del Hospital Manuel Nuñez Butrón, donde, en cuanto a la Dimensión de Seguridad el $70 \%$ de las madres manifiestan satisfacción completa, seguido del $25 \%$ de satisfacción intermedia y finalmente un 5\% de insatisfacción por parte de las madres. 


\section{Tabla 7}

Satisfacción, en la dimensión empatía, de las madres de niños hospitalizados frente a los cuidados de Enfermería en el Servicio de Pediatría del Hospital Regional de Pucallpa, 2020

\begin{tabular}{ccc}
\hline Nivel de Satisfacción en la dimensión empatía & N & $\%$ \\
\hline Satisfacción completa: & 15 & 24.2 \\
Satisfacción intermedia: & 37 & 59.7 \\
Insatisfacción: & 10 & 16.1 \\
Total & 62 & 100.0
\end{tabular}

Fuente: Cuestionario aplicado a las madres de niños Hospitalizados en el servicio de pediatría del HRP, 2020

Del total de madres encuestadas (62), el 59.7\% (37) de ellas expresan satisfacción intermedia; el 24.2\% (15) expresan satisfacción completa; y el 16.1\% (10) de ellas expresan insatisfacción.

Los resultados difieren de los hallazgos de Velásquez D., en el estudio sobre Satisfacción de las madres de niños hospitalizados en pediatría sobre la atención de Enfermería del Hospital Manuel Nuñez Butrón, donde, en cuanto a la Dimensión de Empatía el $65 \%$ de las madres presentan satisfacción completa, seguido del $25 \%$ de satisfacción intermedia y finalmente un 10\% de las madres muestran insatisfacción (13).

\section{CONCLUSIONES}

1. El nivel de satisfacción que predomina es el nivel intermedio con tendencia al nivel de satisfacción completa, pero aún persiste un considerable porcentaje que expresan insatisfacción.

2. La satisfacción, en la dimensión elementos tangibles, el 59.7\% expresaron satisfacción intermedia con tendencia a satisfacción completa. 3. En cuanto a la satisfacción, en la dimensión fiabilidad, 56.5\% expresaron satisfacción intermedia con tendencia a satisfacción completa. 
4. La satisfacción, en la dimensión capacidad de respuesta, el 53.2\% expresaron satisfacción intermedia con tendencia a satisfacción completa. 5. Respecto a la satisfacción, en la dimensión seguridad, el 56.5\% expresaron satisfacción intermedia con tendencia a satisfacción completa.

6. En cuanto a la satisfacción, en la dimensión empatía, el 59.7\% expresaron satisfacción intermedia con tendencia a satisfacción completa.

\section{REFERENCIAS BIBLIOGRÁFICAS}

1. Organización Mundial de la Salud. Boletín: Relación entre la satisfacción con el sistema de atención sanitaria y la experiencia personal de los pacientes. [Internet] Vol $87 \mathrm{f} / \mathrm{p} 2016$.

Consultado 24 de noviembre de 2020. Disponible en: https://www.who.int/bulletin/volumes/87/4/07-050401-ab/es/

2. Organización Panamericana de la Salud. Política y estrategia regional para la garantía de la calidad de la atención sanitaria, incluyendo la seguridad del paciente. Washington, D.C., EUA, 1-5 de octubre del 2017. [Internet]. Consultado 24 de noviembre de 2020. Disponible en: https://www.paho.org/spanish/gov/csp/csp2716-s.pdf

3. Ministerio de Salud. Documento Técnico: Política Nacional de Calidad [sede Web]. Perú: Ministerio de Salud; 2009. Consultado 01 de noviembre de 2020. Disponible en: http://www.minsa.gob.pe/dgsp/archivo/politica_nacional_calidad.pdf

4. Hernández G. El Cuidado de Enfermería: Una Reflexión sobre el Cuidado Humanístico [sede Web]. Chile: Pontificia Universidad Católica de Chile; 2012. Consultado 01 de noviembre de 2020. Disponible en: http://horizonteenfermeria.uc.cl/images/pdf/23-2/editorial.pdf.pdf

5. González A, Padín S, de Ramón E. Satisfacción del paciente con la atención de enfermería. Enfermería Clínica [Internet]. España: sciencedirect; 2005. Consultado 01 de noviembre de 2020. Disponible en: https://www.sciencedirect.com/science/article/pii/S1130862105711013 
6. Caizabanda G. Nivel de satisfacción del usuario y su relación con las acciones del personal de Enfermería para el cuidado del niño del área de Pediatría del HPDA durante el período marzo - agosto 2016. [Internet]. f/p 2016. Universidad Técnica de Ambato. [Tesis de titulación]. Consultado 01 de noviembre de 2020. Disponible en:

https://repositorio.uta.edu.ec/jspui/bitstream/123456789/9471/1/Caizabanda\%20L ema\%2C \%20Gladys\%20Marisol.pdf

7. Moreno M., Interial M., Sauceda P., Vázquez L., y López J. Satisfacción de las madres con la atención a sus hijos hospitalizados. [Internet]. f/p 2016. Universidad de la Sabana - Aquichán. [Tesis de disertación]. Consultado 01 de noviembre de 2020.

Disponible

en:

https://aquichan.unisabana.edu.co/index.php/aquichan/article/view/1816/2383

8. Coronado A., y Vasquez K. Nivel de satisfacción de la madre sobre el cuidado brindado por la enfermera al niño hospitalizado en Pediatría del Hospital Regional Lambayeque, 2017. [Internet]. f/p Chiclayo, 21 de Junio del 2018. Universidad Católica Santo Toribio de Mogrovejo. [Tesis de titulación]. Consultado 02 de noviembre de 2020. Disponible en: http://tesis.usat.edu.pe/bitstream/20.500.12423/1246/1/TL_CoronadoChanameAna Vasque zSantistebanKaren.pdf.pdf

9. Maceda S. Nivel de satisfacción de las madres de niños hospitalizados frente a los cuidados de enfermería en el Servicio de Pediatría Hospital Regional II- 2 Tumbes. 2015. [Internet]. f/p 2015. Universidad Nacional de Tumbes. [Tesis de titulación]. Consultado 02 de noviembre de 2020. Disponible en:

http://repositorio.untumbes.edu.pe/bitstream/handle/UNITUMBES/204/TESIS\%20 $\underline{\mathrm{DE} \% 20}$

MAESTRIA $\% 20-$ \%20SARA\%20MACEDA\%20GUARANDA.pdf?sequence=1\&isAllowed=y

10. Velásquez D. Satisfacción de las madres de niños hospitalizados en pediatría sobre la atención de Enfermería del Hospital Manuel Nuñez Butrón. [Internet]. f/p Puno 2015.

Universidad Nacional del Altiplano. [Tesis de titulación]. Consultado 03 de noviembre de 
en:

http://repositorio.unap.edu.pe/bitstream/handle/UNAP/2291/Velasquez_Titalo_Dia $\underline{\text { na_Este la.pdf? sequence }=1 \& \text { isAllowed }=y}$

11. Alarcón Y, Gómez D. Nivel de satisfacción del paciente adulto mayor sobre el cuidado de enfermería en el Servicio de Medicina del Hospital Regional de Pucallpa y Hospital Amazónico en el mes de Junio y Julio 2017. [Internet]. f/p Pucallpa 2018. Universidad Nacional de Ucayali. [Tesis de titulación]. Consultado 03 de noviembre de 2020. Disponible en: http://repositorio.unu.edu.pe/handle/UNU/3604.

12. Ramírez A., y Tello R. 2017. Nivel de satisfacción del paciente hospitalizado con los cuidados de enfermería en el Hospital II ESSalud-Pucallpa 2016. [Internet]. f/p Pucallpa 2017. Universidad Nacional de Ucayali. [Tesis de titulación]. Consultado 25 de noviembre de 2020. Disponible en: http://repositorio.unu.edu.pe/handle/UNU/3386

13. Dávila M., Lulo A., y Riva J. Calidad del cuidado desde la percepción del paciente que brinda el estudiante de enfermería de la Universidad Nacional de Ucayali, en el hospital regional de

Pucallpa 2014. [Internet]. f/p Pucallpa 2015. Universidad Nacional de Ucayali.

[Tesis de

titulación]. Consultado 26 de noviembre de 2020. Disponible en: https://alicia.concytec.gob.pe/vufind/Record/RUNU_228737750fb2d9d5f86d7d54 bc2f5de

1

14. Panduro K., Rodriguez L., y Panduro K. Satisfacción de la madre sobre la atención de enfermería e influencia en el cumplimiento del número de controles en la estrategia de crecimiento y desarrollo en niños menores de un año; hospital regional de Pucallpa - Ucayali 2013. [Internet]. f/p Pucallpa 2014. Universidad Nacional de Ucayali. [Tesis de titulación]. 
Nicolás Magno Fretel, Eldalaine Torres Vargas, Holger Alex Aranciaga, Roger Habraham Tapia, Reyna Isabel Bastos Vásquez

Consultado 26 de noviembre de 2020. Disponible en:

https://alicia.concytec.gob.pe/vufind/Record/RUNU_96e8f73b741ae7828adfb4901

$\underline{\text { 3a1elcb }}$ /Description\#tabnav

15. Calva J. Satisfacción de usuarios: la investigación sobre las necesidades de información.

[Internet]. Consultado 01 de noviembre de 2020. Disponible en:

http://132.248.242.3/ publica/archivos/libros/satisfaccion_usuarios.pdf

16. Tenopir C. "Calidad en el Ambiente Electrónico". [Internet]. Consultado 04 de noviembre de 2020. Disponible en: https://www.calidad-

gestion.com.ar/boletin/64_sistema_de_gestion_formato_eletronico.html

17. MINSA. Capacitación para la Gestión Enfoques y metodologías y Promoción de la Salud Materno Infantil en REDES y la Dirección de Salud Puno, setiembre del 2018.

18. Blog. Gestión de operaciones. El Modelo SERVQUAL de Calidad de Servicio. GEO Tutoriales el 02/02/2016 en Gestión de Calidad. [Internet]. Consultado 22 de noviembre de 2020. Disponible en: https://www.gestiondeoperaciones.net/gestionde-calidad/elmodeloservqual-de-calidad-de-servicio/

19. Sánchez M. Medición de la calidad en el Servicio. [Internet]. Consultado 04 de noviembre de 2020. Disponible en: https://www.uv.mx/iiesca/files/2017/03/11CA201602.pdf 